\title{
COMMENT
}

\section{Is bronchopulmonary dysplasia decided before birth?}

\author{
Vineet Bhandari ${ }^{1}$ and Abhay Lodha ${ }^{2}$ \\ Pediatric Research (2020) 87:809-810; https://doi.org/10.1038/s41390-020-0819-4
}

Bronchopulmonary dysplasia (BPD) is a complex disorder secondary to genetic-environmental interactions. ${ }^{1-3}$ The complexity in the pathogenesis of this developmental disorder has contributed to the difficulty in developing specific and effective preventative and therapeutic interventions to combat the devastating disease. ${ }^{4}$ The impact of this most common chronic lung disease of infancy extends beyond the neonatal intensive care unit (NICU), up to adulthood, and is a significant contributor to healthcare costs. ${ }^{5,6}$

Among the environmental factors contributing to the pathogenesis of BPD, the three most well established are exposure to invasive mechanical ventilation (MV), hyperoxia, and sepsis. While the first two are post-natal, sepsis (which could be localized to the lung or systemic) can be ante- or post-natal in origin. In the antenatal scenario, the focus has been on the presence of chorioamnionitis. $^{7-9}$ Interestingly, the role of chorioamnionitis (partly due to difficulties in diagnosis) as a contributory factor to BPD remains controversial. ${ }^{7,8,10-12}$ Downstream of all these factors has been the presence of persistent inflammation subverting the normal process of lung development. In the current issue of Pediatric Research, Mir et al. ${ }^{13}$ highlighted the potential role of a variety of placental pathologies and the outcomes of BPD/death and neurodevelopmental impairment (NDI) at 2 years.

In their retrospective study of neonates $<29$ weeks gestation, ${ }^{13}$ the investigators categorized placental lesions into none, 1 , or $\geq 2$ and noted that as the number of placental lesions increased, the outcomes of BPD/death and NDI were significantly increased. After controlling for confounding factors, the association with the presence of moderate-severe BPD (odds ratio [OR]: 3.9; $95 \%$ confidence interval $[\mathrm{Cl}]: 1.5-10 ; p<0.01)$ remained significant, but did not hold for NDI (OR: $1.6 ; 95 \% \mathrm{Cl}: 0.7-3.7 ; p=0.28) .{ }^{13}$ Previous investigators have evaluated different types of placental pathologies with neonatal outcomes; ${ }^{14-25}$ however, the current study is the first one to assess the number of placental pathologic lesions with the neonatal outcomes of mortality, BPD, and NDI.

It is imperative to be aware of some of the limitations of the current study. ${ }^{13}$ There are some well-known shortcomings of a retrospective study, for example, missing or misclassification of data, among others. It is also important to recognize that causality cannot be established definitively in such retrospective epidemiologic studies. This study was not powered for the primary outcome. Moderate-severe BPD was defined solely as the need for supplemental oxygen at 36 weeks post-menstrual age. As the authors have also mentioned, data on clinical severity of illness was not collected. It is difficult to establish based on the study findings that multiple placental pathologies were the only contributing factor to $\mathrm{NDI}$ or was secondary to BPD or a combination of multiple factors during the NICU stay. Prolonged
MV is associated with acute inflammatory changes that might affect outcomes in premature infants. Therefore, one of the confounding factors was the duration of MV and this was not adjusted in the logistic regression model. In addition, unknown variables could be further confounders, and the fact that, despite the separate categorization of the placental pathological lesions, there is an interaction between the vascular and inflammatory pathologies reflecting the severity of each or a combination of the insult/injury.

The five independent significant placental pathologies assessed included acute histologic chorioamnionitis (AHC), high-grade villitis, maternal vascular underperfusion (MVU), fetal thrombotic vasculopathy (FTV), and small or large for gestational age (S/LGA) placentas. Among those with multiple significant placental lesions, the most common combinations were S/LGA or MVU with AHC. Interestingly, no single pathologic lesion was associated with $\mathrm{BPD}^{13}$

As mentioned above, while the association of BPD with the presence of chorioamnionitis remains controversial, ${ }^{7,8,10-12}$ some of the other placental lesions appear to be associated with specific outcomes. For example, two studies have reported MVU/decreased villous vascularity in preterm infants who develop BPD-associated pulmonary hypertension $(\mathrm{PH}){ }^{16,26} \mathrm{~A}$ recent study reported that decreased levels of cord blood angiogenic factors (placental growth factor, granulocyte-colony-stimulating factor, and vascular endothelial growth factor A) were associated with placental MVU and BPD$\mathrm{PH}^{27}$ Antenatal placental vasculopathy predisposing to disordered blood vessels in infants going on to develop BPD-PH might also be suggested with the association of maternal diabetes with BPD-PH, both for early and late $\mathrm{PH}$, as recently reported. ${ }^{28}$ It is certainly exciting to investigate the possibility of antenatal placental vasculopathy as a continuum towards a predisposition for developing early and/or late PH in infants with BPD. ${ }^{29}$ Singlenucleotide polymorphisms of arginase-1 (ARG1 rs2781666), and/or dimethylarginine dimethylaminohydrolase-1 (DDAH1 rs480414), were associated with a decreased risk of developing of BPD-PH. ${ }^{30,31}$

Thus, to answer the question posed in the title, probably not, but the antenatal origins of the possibility of BPD-PH warrants active investigation. Concerted efforts to understand the pathogenesis of BPD-PH will certainly pave dividends for an earlier identification and improved therapeutic options for a condition that has such a high morbidity and mortality. The association between BPD and the presence of various placental pathologies such as MVU, FTV, chronic villitis, villous edema, and acute chorioamnionitis has been inconsistent and has not been studied prospectively in a well-powered study. Such studies could allow physicians in NICUs to utilize the findings of placental histopathology to help counsel parents about the possibility of

\footnotetext{
'Department of Pediatrics, Division of Neonatology, The Children's Regional Hospital at Cooper, Cooper Medical School at Rowan University, Camden, NJ, USA and ${ }^{2}$ Section of Neonatology, Department of Pediatrics, University of Calgary, Alberta Children's Hospital Research Institute, University of Calgary, Calgary, AB, Canada Correspondence: Vineet Bhandari (bhandari-vineet@cooperhealth.edu)
}

Received: 25 December 2019 Accepted: 8 February 2020

Published online: 17 February 2020 
early prediction of BPD and possibly, its association for NDI, if found to be true.

\section{AUTHOR CONTRIBUTIONS}

V.B. wrote the first draft. A.L. edited the manuscript and provided additional intellectual content. Both authors approved the final draft of the manuscript.

\section{ADDITIONAL INFORMATION}

Competing interests: The authors declare no competing interests.

Publisher's note Springer Nature remains neutral with regard to jurisdictional claims in published maps and institutional affiliations.

\section{REFERENCES}

1. Bhandari, V. Designing a better definition of bronchopulmonary dysplasia. Pediatr. Pulmonol. 54, 678-679 (2019).

2. Lal, C. V., Bhandari, V. \& Ambalavanan, N. Genomics, microbiomics, proteomics, and metabolomics in bronchopulmonary dysplasia. Semin. Perinatol. 42, 425-431 (2018).

3. Philpot, P. A. \& Bhandari, V. Predicting the likelihood of bronchopulmonary dysplasia in premature neonates. Expert Rev. Respir. Med. 13, 871-884 (2019).

4. Bhandari, V. Drug therapy trials for the prevention of bronchopulmonary dysplasia: current and future targets. Front. Pediatr. 2, 76 (2014).

5. Lapcharoensap, W., Bennett, M. V., Xu, X., Lee, H. C. \& Dukhovny, D. Hospitalization costs associated with bronchopulmonary dysplasia in the first year of life. J. Perinatol. 40, 130-137 (2020).

6. Alvarez-Fuente, M. et al. The economic impact of prematurity and bronchopulmonary dysplasia. Eur. J. Pediatr. 176, 1587-1593 (2017).

7. Sarno, L. et al. Histological chorioamnionitis and risk of pulmonary complications in preterm births: a systematic review and meta-analysis. J. Matern. Fetal Neonatal Med. 1-10 (2019). https://doi.org/10.1080/14767058.2019.1689945. [Epub ahead of print].

8. Villamor-Martinez, E. et al. Association of chorioamnionitis with bronchopulmonary dysplasia among preterm infants: a systematic review, meta-analysis, and metaregression. JAMA Netw. Open 2, e1914611 (2019).

9. Bashir, R. A. et al. Chorioamnionitis at birth does not increase the risk of neurodevelopmental disability in premature infants with bronchopulmonary dysplasia. Acta Paediatr. 105, e506-e512 (2016).

10. Ericson, J. E. \& Laughon, M. M. Chorioamnionitis: implications for the neonate. Clin. Perinatol. 42, 155-165 (2015). ix.

11. Thomas, W. \& Speer, C. P. Chorioamnionitis is essential in the evolution of bronchopulmonary dysplasia-the case in favour. Paediatr. Respir. Rev. 15, 49-52 (2014).

12. Lacaze-Masmonteil, T. That chorioamnionitis is a risk factor for bronchopulmonary dysplasia-the case against. Paediatr. Respir. Rev. 15, 53-55 (2014).

13. Mir, I. N., et al. Impact of multiple placental pathologies on neonatal death, bronchopulmonary dysplasia, and neurodevelopmental impairment in preterm infants. Pediatr. Res. (2019). https://doi.org/10.1038/s41390-019-0715-y. [Epub ahead of print].
14. Dhatariya, K. K. et al. The cost of treating diabetic ketoacidosis in an adolescent population in the UK: a national survey of hospital resource use. Diabet. Med. 36, 982-987 (2019).

15. Strouss, L., Goldstein, N. D., Locke, R. \& Paul, D. A. Vascular placental pathology and the relationship between hypertensive disorders of pregnancy and neonatal outcomes in very low birth weight infants. J. Perinatol. 38, 324-331 (2018).

16. Kunjunju, A. M., Gopagondanahalli, K. R., Chan, Y. \& Sehgal, A. Bronchopulmonary dysplasia-associated pulmonary hypertension: clues from placental pathology. J. Perinatol. 37, 1310-1314 (2017).

17. Chisholm, K. M. et al. Correlation of preterm infant illness severity with placental histology. Placenta 39, 61-69 (2016)

18. Vinnars, M. T., Papadogiannakis, N., Nasiell, J., Holmstrom, G. \& Westgren, M. Placental pathology in relation to stillbirth and neonatal outcome in an extremely preterm population: a prospective cohort study. Acta Obstet. Gynecol. Scand. 94, 584-590 (2015)

19. Mestan, K. K. et al. Placental pathologic changes of maternal vascular underperfusion in bronchopulmonary dysplasia and pulmonary hypertension. Placenta 35, 570-574 (2014).

20. Vinnars, M. T. et al. Association between placental pathology and neonatal outcome in preeclampsia: a large cohort study. Hypertens. Pregnancy 33, 145-158 (2014).

21. Perrone, S. et al. Perinatal outcome and placental histological characteristics: a single-center study. J. Matern. Fetal Neonatal Med. 25(Suppl 1), 110-113 (2012).

22. Mehta, R., Nanjundaswamy, S., Shen-Schwarz, S. \& Petrova, A. Neonatal morbidity and placental pathology. Indian J. Pediatr. 73, 25-28 (2006).

23. Ogunyemi, D., Murillo, M., Jackson, U., Hunter, N. \& Alperson, B. The relationship between placental histopathology findings and perinatal outcome in preterm infants. J. Matern. Fetal Neonatal Med. 13, 102-109 (2003).

24. Pendleton, R. G., Kaiser, C. \& Gessner, G. Studies on adrenal phenylethanolamine $\mathrm{N}$-methyltransferase (PNMT) with S K \& F 64139, a selective inhibitor. J. Pharm. Exp. Ther. 197, 623-632 (1976).

25. Rude, E., Wrede, J. \& Gundelach, M. L. Production of IgG antibodies and enhanced response of nude mice to DNP-AE-dextran. J. Immunol. 116, 527-533 (1976).

26. Yallapragada, S. G. et al. Placental villous vascularity is decreased in premature infants with bronchopulmonary dysplasia-associated pulmonary hypertension. Pediatr. Dev. Pathol. 19, 101-107 (2016).

27. Mestan, K. K. et al. Cord blood biomarkers of placental maternal vascular underperfusion predict bronchopulmonary dysplasia-associated pulmonary hypertension. J. Pediatr. 185, 33-41 (2017).

28. Sheth, S., Goto, L., Bhandari, V., Abraham, B. \& Mowes, A. Factors associated with development of early and late pulmonary hypertension in preterm infants with bronchopulmonary dysplasia. J. Perinatol. 40, 138-148 (2020).

29. Taglauer, E., Abman, S. H. \& Keller, R. L. Recent advances in antenatal factors predisposing to bronchopulmonary dysplasia. Semin. Perinatol. 42, 413-424 (2018).

30. Trittmann, J. K. et al. Using clinical and genetic data to predict pulmonary hypertension in bronchopulmonary dysplasia. Acta Paediatr. 107, 2158-2164 (2018).

31. Trittmann, J. K. et al. Arginase I gene single-nucleotide polymorphism is associated with decreased risk of pulmonary hypertension in bronchopulmonary dysplasia. Acta Paediatr. 103, e439-e443 (2014). 